\title{
Tuberculosis extrapulmonar en nuestro medio. Formas de presentación
}

\author{
F.L. LADO LADO, V. TUÑEZ BASTIDA**, A.L. GOLPE GÓMEZ*, M.J. FERREIRO \\ REGUEIRO, A. CABARCOS ORTIZ DE BARRÓN
}

Servicios de Medicina Interna y *Neumología. Complejo Hospitalario Universitario. Santiago. Departamento de Medicina. **Unidad de Prevención y Control de Tuberculosis. Área Sanitaria. Santiago de Compostela.

\author{
EXTRAPULMONARY TUBERCULOSIS IN OUR AREA. FORMS OF \\ PRESENTATION
}

\begin{abstract}
RESUMEN
Objetivo: Analizar la distribución de las formas de presentación de tuberculosis extrapulmonar (FPE) en nuestro medio.

Material y métodos: Se revisaron los casos de tuberculosis extrapulmonar registrados en la Unidad de Prevención y Control de la Tuberculosis del Área Sanitaria de Santiago de Compostela en un periodo de tres años. Clasificamos las FPE en: formas extrapulmonares (FE), definida por una localización extrapulmonar; formas mixtas (FM), localización pulmonar y extrapulmonar; formas diseminadas (FD), dos o más localizaciones extrapulmonares; y TB miliares, definida por patrón radiológico miliar y/o enfermedad diseminada en necropsia.

Resultados: Se registraron 921 tuberculosis de las cuales 370 $(40,2 \%)$ fueron extrapulmonares. De ellos 199 eran varones y 171 mujeres. Edad media de 36,9 años (rango, 19 y 68). Se constató infección por el virus de la inmunodeficiencia humana (VIH) en 22 casos $(25,9 \%)$. La distribución de las FPE fue: FE 307 casos $(83,0 \%)$ : pleural $140(45,6 \%)$, ganglionar $87(28,3 \%)$, intestinal $16(5,2 \%)$, osteoarticular $14(4,5 \%)$, genitourinaria $11(3,6 \%)$, cutánea $11(3,6 \%)$, meníngea $10(3,3 \%)$, otras $18(5,9 \%)$; FM 38 (10,3\%); FD $8(2,1 \%)$ y TB miliares 17 casos $(4,6 \%)$. Los pacientes con infección por el VIH presentaron FE en 17 casos $(77,3 \%)$ en su mayoría ganglionar $(64,7 \%) ; \mathrm{FD}$ en $4(18,2 \%)$; у TB miliar en $1(4,5 \%)$.

Conclusiones: La tuberculosis extrapulmonar supera las expectativas esperables, no en vano refleja un porcentaje próximo a la localización pulmonar. Ello indica por una parte, un cambio significativo en la presentación clásica de la enfermedad, y por otra una extrema sensibilidad en el diagnóstico localizatorio de la enfermedad.
\end{abstract}

PALABRAS CLAVE: Tuberculosis extrapulmonar. Infección por VIH.

\section{ABSTRACT}

Objetive: To analyse the distribution of the extrapulmonary forms of presentation of tuberculosis $(E F P)$ in our health district.

Materials and methods: The medical records of patients diagnosed with extrapulmonary tuberculosis attending the Tuberculosis Prevention and Control Unit of the Santiago Health District were reviewed over a three years period. The classification of EFP was: extrapulmonary forms $(E F)$, disease outside the lung; mixed forms $(M F)$, the presence of both pulmonary and extrapulmonary tuberculosis; disseminated forms $(D F)$, the pressence of two or more extrapulmonary locations; and miliary $T B$, which was definied by a diffuse pulmonary radiographic pattern or diag nosis was undertaken by necropsy.

Results: A total of 921 tuberculosis infected patients were observed, of which $370(40.2 \%)$ were extrapulmonary forms; of these, 199 were men and 171 women (mean age was 36.9 years, range 19-68). The distri bution of EFP was: 307 EF (83\%) of which 140 (45.6\%) were pleural, $87(28.3 \%)$ ganglionary, $16(5.2 \%)$ intestinal, $14(4.5 \%)$ bone and joint, $11(3.6 \%)$ genitourinary, 11 (3.6\%) cutaneous, 10 (3.3\%) meningeal, and other locations 18 (5.9); MF 38 cases (10.3\%); DF 8 cases $(2.1 \%)$ and miliary TB 1 cases (4.6\%). In HIV infected patients 17 EF (77.3\%), which were mainly ganglionary (64.7\%); $4 \mathrm{DF}$ (18.2\%); and 1miliary $T B(4.5 \%)$ cases were observed.

Conclusions: Our findings confirm the high incidence of extrapulmo nary $T B$, similar to the number of pulmonary forms. Our experience shows a significant modification to the classical presentation of the disease, and thus the need for sensitivity in locating the disease.

KEY WORDS: Tuberculosis extrapulmonary. HIV.

Lado Lado FL, Túñez Bastida V, Golpe Gómez AL, Ferreiro Regueiro MJ, Cabarcos Ortiz de Barrón A. Tuberculosis extrapulmonar en nuestro medio. Formas de presentación. An Med Interna (Madrid) 2000; 17: 637-641.

\section{INTRODUCCIÓN}

En el momento actual, la tuberculosis sigue siendo un problema sociosanitario relevante incluso en países desarrollados. Diversos factores han sido implicados, entre ellos, la irrupción de la infección producida por el virus de la inmunodeficiencia humana (VIH) que ha dado lugar a modificaciones en todos los aspectos conocidos de la tuberculosis, tanto en la epidemiología, patogénesis, diagnóstico, profilaxis, tratamiento y la presentación de la infección por el bacilo tuberculoso, como en la expresividad clínica de la enfermedad tuberculosa (1-8).

Los cambios demográficos y los integrados por ciertos grupos marginales (como los indigentes) y los hábitos tóxi-

Trabajo aceptado: 15 de Junio de 2000

Correspondencia: F.L. Lado Lado. Servicio de Medicina Interna. Hospital Gil Casares C/A Choupana s/n 15706 Santiago de Compostela. A Coruña. 
cos (la drogadicción y el alcoholismo) han sido factores influyentes que, en conjunto, han contribuído a un descenso del control de la tuberculosis (9-13). Asimismo, la eclosión de brotes en instituciones cerradas y la aparición de resistencias a los tratamientos habituales han suscitado una preocupación extrema en el control de la enfermedad y en la búsqueda de métodos de diagnóstico rápido y de nuevas alternativas terapeúticas para el tratamiento de esta enfermedad (14-20).

En la población inmunocompetente, la forma de presentación habitual de la tuberculosis es la pulmonar. Así, en el año 1986 de 22.764 casos declarados en EE.UU. el 82,5\% eran pulmonares mientras que el $17,5 \%$ restante tenían presentación extrapulmonar, representadas principalmente por las localizaciones ganglionar, pleural y génitourinaria (21). Sin embargo, otros autores (22-24), en estudios realizados en el ámbito nacional, refieren una frecuencia superior $(27,5-37 \%)$ a la indicada de tuberculosis extrapulmonar con predominio de la localización pleural. En los pacientes infectados por el VIH, la tuberculosis extrapulmonar adquirió tal importancia que en el año 1987 se incluyó como enfermedad definitoria de SIDA (25).

El objetivo del presente estudio es conocer la distribución de las distintas formas de presentación de la enfermedad tuberculosa extrapulmonar (FPE) en nuestro medio, a partir de los casos registrados en una unidad de prevención y control de tuberculosis durante un período de tres años.

\section{MATERIAL Y MÉTODOS}

Se trata de un análisis retrospectivo en el que se revisan los casos de enfermedad tuberculosa registrados en la Unidad de Prevención y Control de la Tuberculosis del Área Sanitaria de Santiago de Compostela que abarca una población urbana y rural estimada en aproximadamente 500.000 habitantes. El período de estudio comprende entre noviembre de 1994 y Noviembre de 1997.

En el presente estudio se incluyeron todos aquellos casos diagnosticados de tuberculosis extrapulmonar -con introducción de los casos en los que, concomitantemente, se constató participación pulmonar- mediante los criterios que detallamos a continuación:

-Presencia de bacilos ácido alcohol resistentes (BAAR) mediante la tinción de Ziehl-Nielsen o de auramina-rodamina y/o cultivo positivo para Mycobacterium tuberculosis en medio de Löwestein-Jensen en una o más muestras biológicas.

-Evidencia de granulomas con necrosis caseosa en los estudio histológicos procedentes de las distintas muestras obtenidas por punción aspiración con aguja fina (PAAF) o por biopsia.

-Derrame pleural con características de exudado de predominio linfocitario que tenían adenosindeaminasa (ADA) superior a $47 \mathrm{UI} / \mathrm{ml}$ (26), y datos clínicos compatibles además de PPD positiva, con respuesta al tratamiento tuberculostático, tras haberse descartado otras posibilidades diagnósticas.

Se recogieron como datos referentes a factores predisponentes los relativos a los antecedentes conocidos de alcoholismo o de drogadicción, estado serológico frente al VIH y antecedentes de entidades y/o procesos que, en su curso, tuvieran implícitos datos de inmunosupresión tales como: insuficiencia renal crónica, neoplasias, tratamiento prolongado con corticoides, hipogammaglobulinemia y enfermedades del tejido conectivo; y otros como: enfermedad pulmonar obstructiva crónica y diabetes mellitus.

Se clasificaron las distintas formas de presentación de la tuberculosis extrapulmonar (FPE) en las siguientes categorías: Formas extrapulmonares (FE), definida por una única localización extrapulmonar; formas mixtas (FM), localización pulmonar y extrapulmonar; formas diseminadas (FD), dos o más localizaciones extrapulmonares; y TB miliares, definidas por patrón miliar en la radiografía de tórax y/o enfermedad diseminada en necropsia.

En el análisis estadístico se introdujeron los datos en hoja de cálculo Excel (ver 97). Se emplearon las medias, porcentajes y comparación de proporciones.

\section{RESULTADOS}

En el periodo descrito, se registraron un total de 921 casos de tuberculosis de los cuales $370(40,2 \%)$ se corresponden con tuberculosis de localización extrapulmonar. De los 370 casos de tuberculosis extrapulmonar estudiados, 199 eran varones $(53,8 \%)$ y 171 mujeres $(46,2 \%)$, con una edad media de 36,9 años y rango entre 19 y 68 . Un total de 85 casos $(23,0 \%)$ presentaba algún factor predisponente, ya fuera de forma aislada o combinada, los más comunes fueron: alcoholismo en 24 casos $(28,2 \%)$, infección por el virus de la inmunodeficiencia humana (VIH) en 22 $(25,9 \%)$, inmunodepresión en $21(24,7 \%)$, usuarios a drogas por vía parenteral en $13(15,3 \%)$, y otras en 5 casos $(5,9 \%)$.

La distribución de las distintas FPE fue: FE resultaron un total 307 casos $(83,0 \%)$ que se distribuyeron como sigue: Pleural 140 (45,6\%), Ganglionar 87 (28,3\%), Intestinal 16 $(5,2 \%)$, Osteoarticular $14(4,5 \%)$, Genitourinaria $11(3,6 \%)$, Cutánea 11 (3,6\%), Meníngea 10 (3,3\%), Otras 18 (5,9\%) que se distribuyeron como sigue: pericárdica en 9 casos, área ORL en 6 , ocular peritonela y hepática en 1 respectivamente. FM resulatron un total de 38 casos $(10,3 \%)$, TB miliares un total de $17(4,6 \%)$ siendo dos casos diagnosticados en necropsia y FD 8 casos $(2,1 \%)$ (Figs. 1 y 2 ).

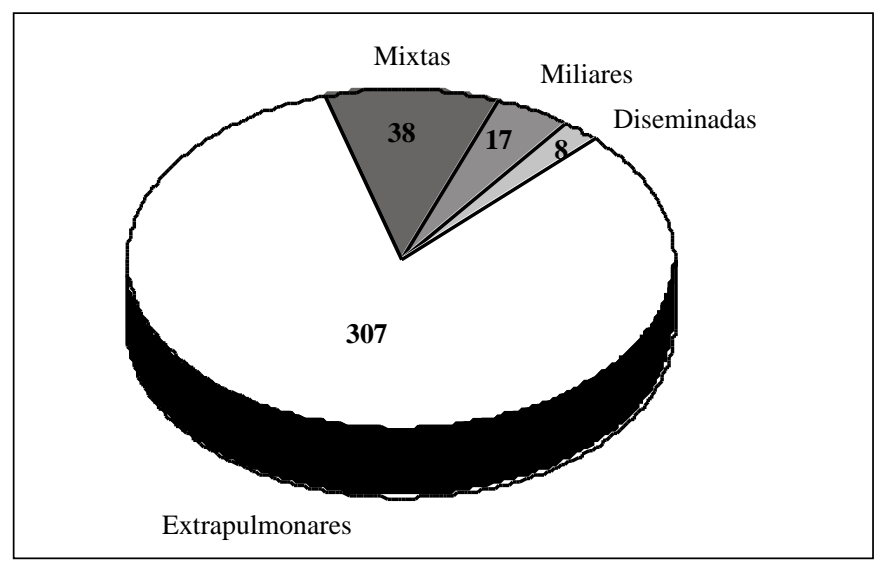

Fig. 1. Prevalencia de las distintas formas de presentación extrapulmonar. 


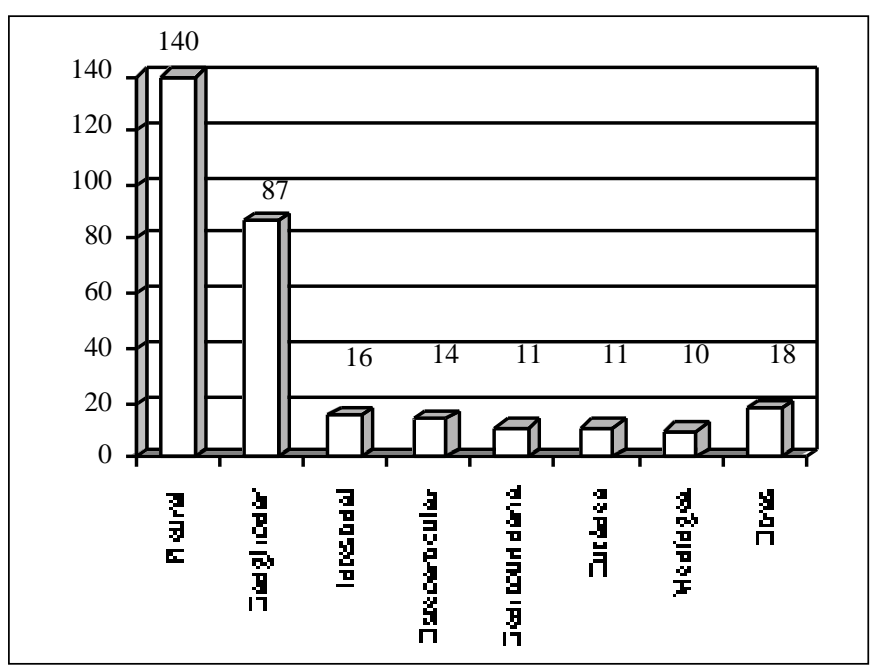

Fig. 2. Distribución de las formas extrapulmonares.

En los pacientes con serología positiva para el VIH se presentaron FE en un total de 17 casos $(77,3 \%)$ siendo en su mayoría localización ganglionar declarándose un total de 11 casos $(64,7 \%)$, a continuación localización intestinal y osteoarticular en 2 respectivamente y, por último, localización ótica y meníngea en un caso respectivamente; las formas mixtas, diseminadas y tuberculosis miliares en estos pacientes representaron un total de 5 casos $(22,7 \%)$ (Tabla I).

En cuanto, a la distribución por edades de las FE más frecuentes, se detectó que en 53 casos $(37,8 \%)$ la presentación Pleural aconteció entre los 15 y 25 años de edad, mientras que en la Ganglionar 14 casos $(16,1 \%)$ ocurrieron en el mencionado grupo de edad y $9(10,3 \%)$ por debajo de los 15 años (Tabla II).

\section{DISCUSIÓN}

En nuestro medio, la tuberculosis se caracteriza por tener una incidencia elevada. Así entre los años 1989 y 1994 osciló entre 65,8 y 95,5 casos por 100.000 habitantes $(27,28)$. En el año 1995 se registró una incidencia de 67,4 casos por 100.000 habitantes (29). Por tal motivo, a nivel sociosanitario existe un alto grado de preocupación en lo que supone un amplio cono-

\section{TABLA I}

FPE DECLARADAS SEGÚN ESTADO DE SERO PO SITIVAD O NO FRENTE AL VIH

\begin{tabular}{lcccc}
\hline FPE & $\mathrm{N}$ & VIH+ & VIH- & Significación \\
\hline Extrapulmonar & 307 & 17 & 290 & NS \\
$\quad$ Pleural & 140 & 0 & 140 & $<0,001$ \\
Ganglionar & 87 & 11 & 76 & $<0,01$ \\
Mixtas & 38 & 0 & 38 & $<0,01$ \\
Diseminada & 8 & 4 & 4 & $<0,05$ \\
Miliar & 17 & 1 & 16 & NS \\
Total & 370 & 22 & 348 &
\end{tabular}

FPE: formas de presentación extrapulmonar de la tuberculosis.
TABLA II

EDAD M EDIA Y RANGOS EN LAS DISTINTAS FPE OBJETO DE ESTUDIO

\begin{tabular}{lccc}
\hline Localización & $\mathrm{N}$ & Edad media & Rango \\
\hline Pleural & 140 & 33,1 & $3-87$ \\
Ganglionar & 87 & 35,1 & $2-79$ \\
Intestinal & 16 & 53,1 & $30-67$ \\
O steoarticular & 14 & 60,1 & $23-86$ \\
Génitourinaria & 11 & 35,8 & $16-68$ \\
Cutánea & 11 & 58,0 & $21-67$ \\
M eníngea & 10 & 44,4 & $14-87$ \\
Otras & 18 & 41,9 & $1-75$ \\
Diseminada & 8 & 43,5 & $21-85$ \\
Miliar & 17 & 54,5 & $31-78$ \\
Mixta & 38 & 37,2 & $1-85$ \\
\hline
\end{tabular}

FPE: formas de presentación extrapulmonar de la tuberculosis.

cimiento y estrecho control sobre la repercusión de la enfermedad tuberculosa (30).

En el presente estudio, la localización extrapulmonar supone el 40,2\% del total de tuberculosis declaradas con frecuencia similar a la descrita por otros autores (31), ello contrasta con el hecho de que, clásicamente, dicha localización venía a representar una estimación inferior al $20 \%$ en la población inmunocompetente (21). Tal incremento nos hace pensar que en los últimos años el comportamiento de la tuberculosis está cambiando, habida cuenta que en la presente serie se constató infección por el VIH sólo en el 5,9\% de los casos. En este sentido, es sabido que, en estos pacientes la presentación clínica de la tuberculosis tiene notables diferencias resumidas en una mayor proporción de formas atípicas y diseminadas de la enfermedad, especialmente en las fases avanzadas de inmunodeficiencia, siendo mucho más frecuentes las localizaciones extrapulmonares $(7,32)$.

Los factores predisponentes de relevancia resultaron ser, al igual que en otras series $(22,24)$ y entre otros, el alcoholismo, la infección por el VIH y la drogadicción; sin embargo, no coincidimos en cuanto a su frecuencia, sensiblemente superior $(52,2 \%)$ a la observada en nuestro estudio y ello, en parte, puede ser debido a que se analizan otros factores predisponentes como son la estancia previa en prisión y la inmigración (24).

Con respecto a las formas extrapulmonares, y de forma similar a otros autores, (22-24) encontramos como localizaciones más frecuentes $(73,9 \%)$ la pleural y la ganglionar predominando la primera, por el contrario, otros como Rieder et al (21) hallan más prevalente la localización ganglionar. No obstante, en los pacientes infectados por el VIH, como era de esperar $(33,34)$, la localización extrapulmonar ganglionar fue la más frecuente mientras que no registramos ningún caso de localización pleural, hecho ya constatado por nosotros en otros trabajos en los que encontramos una baja incidencia (34).

Al analizar la distribución por edades de las formas extrapulmonares más prevalentes, como es sabido, la pleuritis tuberculosa puede acontecer a cualquier edad pero es más frecuente (60\%) en grupos inferiores a los 30 años (35), y así lo constatamos en la presente muestra, en la que casi la mitad de 
los casos sucedieron en una población por debajo de los 25 años. En lo que respecta a la localización ganglionar se considera más habitual en grupos de edad inferiores a los 65 años (21), en esta serie la encontramos en casi un tercio de los casos en grupos de edad por debajo de los 25 años. En los pacientes infectados por el VIH, dicha localización, además de ser la forma extrapulmonar más frecuente, representa un grupo muy especial, ya que hemos comprobado en otros estudios que estos pacientes tienen un grado de inmunodeficiencia (poblaciones linfocitarias CD4, respuesta a los tests cutáneos) variable según la expresividad clínica de dicha localización se acompañe o no de otras como la pulmonar (32).

En cuanto a las formas mixtas, que incluyen participación pulmonar y extrapulmonar, resultaron las más frecuentes después de las formas de localización única extrapulmonar con una representación del 10,3\% de los casos, similar a otros autores (22). También es interesante destacar que, aunque no tuvimos ningún caso en los pacientes infectados por el VIH, en nuestra experiencia dicha asociación representa, después de las formas limitadas al pulmón, la observada con mayor frecuencia (8).

Las formas diseminadas, al igual que otros autores (36) incluyen aquellos casos de más de una localización extrapulmonar con el fin de separarlos de aquellos considerados como tuberculosis miliar. En este apartado merece especial atención que observamos una proporción similar tanto en seronegativos como seropositivos frente a la infección por el VIH a pesar de que, en estos últimos, los casos analizados son notablemente menos representativos con respecto a los primeros.
En el grupo restante objeto de análisis, encontramos la tuberculosis miliar, en tercer lugar, después de las formas mixtas con un total de 17 casos $(5 \%)$, resultando más evidente en la población en general (con un total de 16 casos en pacientes seronegativos frente al VIH) que en la de VIH+, donde tan sólo hallamos 1 caso. Tal vez no se refleje fielmente su frecuencia con respecto a las demás formas ya que, en los últimos años, se ha apreciado una mayor incidencia de la tuberculosis miliar en edades avanzadas y pacientes inmunodeprimidos con presentación clínica atípica, que implican una extrema dificultad diagnóstica si no se sospecha la enfermedad y, de hecho, un porcentaje nada despreciable de casos son diagnosticados en necropsia (37-39).

Como resumen de lo expuesto, comentamos que las FPE más frecuentemente registradas han sido las formas extrapulmonares seguidas de las formas mixtas. En las formas extrapulmonares la localización constatada con mayor reiteración ha sido la pleural, seguida de la ganglionar. Los pacientes con serología positiva para el VIH presentaron, con mayor frecuencia, formas extrapulmonares, siendo la localización más común la ganglionar.

Para finalizar, destacamos que la presentación extrapulmonar de la tuberculosis, en nuestro medio, supera las expectativas esperables, y no en vano refleja un porcentaje próximo a la localización pulmonar. Ello nos indica por una parte, un cambio significativo en la presentación clásica de la enfermedad y, por otra una extrema sensibilidad en cuanto al diagnóstico localizatorio de la enfermedad.

\section{Bibliografía}

1. Raviglione MC, Snider DE, Kochi A. Global epidemiology of tuberculosis. Morbidity and mortality of a worldwide epidemic. JAMA 1995; 273: 220-26.

2. Shafer RW, Edlin BR. Tuberculosis in patients infected with immnuodeficency virus: perspective on the past decade. Clin Infect Dis 1996; 22: 683-704.

3. Murray JF. Tuberculosis and HIV infection: global perspectives. Respirology 1997; 3: 209-13.

4. Martínez Vazquez JM, Cabarcos Ortíz de Barrón A, Barrio Gómez E. Tuberculosis e infección por VIH: epidemiología (primera de tres partes). An Med Interna (Madrid) 1997: 14: 253-6.

5. Martínez Vázquez JM, Cabarcos Ortíz de Barrón A, Barrio Gómez E. Tuberculosis e infección por VIH: patogenia (segunda de tres partes). An Med Interna (Madrid) 1997: 14: 310-6.

6. Martínez Vázquez JM, Cabarcos Ortíz de Barrón A, Barrio Gómez E. Tuberculosis e infección por VIH: de la clínica a la prevención (tercera de tres partes). An Med Interna (Madrid) 1997: 14: 363-8.

7. Lado Lado FL, Barrio Gómez E, Carballo Arceo E, Cabarcos Ortíz de Barrón A. Pulmonary tuberculosis with normal radiographs in HIVimmunodeficient patients. AIDS 1999; 13: 1146-7.

8. Lado Lado FL, Barrio Gómez E, Carballo Arceo E, Cabarcos Ortíz de Barrón A. Tuberculosis e infección por el virus de la inmunodeficencia humana: manifestaciones clínicas y rendimiento de procedimientos diagnósticos según las distintas formas de localización de la enfermedad. An Med Interna (Madrid) 2000; 17: 13-8.

9. Brudney K, Dobkin J. Resurgent tuberculosis in New York City. Human immunodeficiency virus, homelessness, and the decline of tuberculosis control programs. Am Rev Respir Dis 1991; 144: 745-9.

10. Cantwell MF, Snider DE, Cauthen GM, Onorato IM. Epidemiology of tuberculosis in the United States, 1985 through 1992. JAMA 1994; 272: 535-9.

11. Zolopa AR, Hahn JA, Gorter R, Miranda J, Wlodarczyk D, Peterson J, et al. HIV and tuberculosis infection in San Francisco's homeless adults. Prevalence and risk factors in a representative sample. JAMA 1994; 272: 455-61.

12. Del Rey Calero. Incremento actual de la tuberculosis. An Med Interna (Madrid) 1995; 12: 209-11.

13. Pablos-Méndez A, Knirsch ChA, Barr RG, Lerner BH, Frieden ThR. Nonadherence in tuberculosis treatment: predictors and consequences in New York city. Am J Med 1997;102:164-70.

14. Fischl MA, Uttamchandani RB, Daikos GL, Poblete RB, Moreno JN, Reyes RR et al. An outbreak of tuberculosis caused by multiple-drugresistant tubercle bacilli among patients with HIV infection. Ann Intern Med 1992; 117: 177-83.

15. Small PM, Shafer RW, Hopewell PC et al. Exogenous reinfection with multidrug-resistant Mycobacterium Tuberculosis in patients with advanced HIV infection. N Engl J Med 1993; 328: 1137-44.

16. De March Ayuela P. La transmisión de la resistencia al Mycobacterium tuberculosis en los infectados por el VIH. La llamada tercera epidemia del VIH. Med Clin (Barc) 1994; 102: 98-100.

17. Chaisson RE, Clermont HC, Holt EA, Cantave M, Johnson MP, Atkinson $\mathrm{J}$ et al. Six-month supervised intermittent tuberculosis therapy in Haitian patients with and without HIV infection. Am J Respir Crit Care Med 1996; 154: 1034-8.

18. Grupo de estudio de tuberculosis resistente de Madrid. Estudio transversal multihospitalario de tuberculosis y resistencias en Madrid. Med Clin (Barc) 1996; 106: 1-6.

19. García Rodríguez JF, Aguado García JM. Tratamiento de la tuberculosis. Ayer, hoy... y mañana. Med Clin (Barc) 1997; 108: 389-95.

20. Gómez Garcés JL. El diagnóstico de la tuberculosis. De rerum harmonicarum entre la clínica y el laboratorio. Rev Clin Esp 1997; 197: 1447. 
21. Rieder HL, Snider DE, Cauthen GM. Extrapulmonary tuberculosis in the United States. Am Rev Respir Dis 1990; 141: 347-51.

22. Fernández Jorge MA, Alonso Mallo E, Lobato Delgado LA, Martínez Sánchez JM. Tuberculosis extrapulmonar: estudio retrospectivo de 107 casos. An Med Interna (Madrid) 1995; 12: 212-5.

23. Caminero JA, Rodríguez de Castro F, Alonso JL, Daryanany RD, Carrillo T, Cabrera P. Epidemiología de la enfermedad tuberculosa en la isla de Gran Canaria. Med Clin (Barc) 1991; 97: 8-13.

24. García Ordóñez MA, Colmenero JD, Valencia A, Pérez Frías J, Sánchez González J, Orihuela F, Causse M, Juárez C. Incidencia y espectro clínico actual de la tuberculosis en un área sanitaria metropolitana del sur de España. Med Clin (Barc) 1998; 110: 51-5.

25. Centers for Disease Control. Revision of the CDC surveillance case definitions for adquired immunodeficiency syndrome. MMWR 1987; 36: $15-55$.

26. Valdés L, Álvarez D, San José E, Juanatey JRG, Pose A, Valle JM, Salgueiro M, Suárez JRR. Value of adenosine deaminase in the diagnosis of tuberculosis pleural effusions in young patients in a region of high prevalence of tuberculosis. Thorax 1995; 50: 600-3.

27. Salgueiro Rodríguez M, Zamarrón Sanz C, Alvarez-Calderón Prat P et al. Estudio epidemiológico de la tuberculosis en el área sanitaria de Santiago de Compostela durante los años 1989, 1990 y 1991. An Med Interna (Madrid) 1993; 10: 427-32.

28. Salgueiro M, Zamarrón C, Otero Y et al. Estudio epidemiológico de la tuberculosis en el área sanitaria de Santiago de Compostela durante 1992,1993 y 1994. An Med Interna (Madrid) 1996; 13: 111-4.

29. Tuñez Bastida MV, Golpe Gómez AL, Paniagua López J. La enfermedad tuberculosa en el área sanitaria de Santiago durante el año 1995. Arch Bronconeumol 1997; 33 (Suppl 1): S32.

30. Anibarro García L, Vázquez-Gallardo R, Toubes Navarro ME, Penas Truque A, Lema Mougán R, Túñez Bastida V et al. Epidemiología de la tuberculosis en Galicia. An Med Interna (Madrid) 1999; 16: 290-6.

31. Cowie RL, Sharpe JW. Extra-pulmonary tuberculosis: a high frecuency in the absence of HIV infection. Int J Tuberc Lung Dis 1997; 2: 159-62.

32. Lado Lado FL, Barrio Gómez E, Carballo Arceo E, Cabarcos Ortíz de Barrón A. Clinical presentation of tuberculosis and the degree of inmunodeficiency in patients with HIV infection. Scand J Infect Dis 1999; 31: 387-91.

33. Shafer RW, Kim DS, Weiss JP, Quale JM. Extrapulmonary tuberculosis in patients with immunodeficiency virus infection. Medicine 1991; 70: 384-97.

34. Lado Lado FL, Barrio Gómez E, Cabarcos Ortíz de Barrón A, Carballo Arceo E, Sánchez Leira J, Pérez del Molino ML, Antúnez López JR. Tuberculosis e infección por el virus de la inmunodeficencia humana. Presentación clínica y diagnóstico. An Med Interna (Madrid) 1998; 15: 415-20.

35. Haro M, Ruiz-Manzano J, Gallego M, Abad J, Manterola JM, Morera J. Tuberculosis pleural: análisis de 105 casos. Enferm Infecc Microbiol Clin 1996; 14: 285-9.

36. Hill AR, Premkumar S, Brustein S et al. Disseminated tuberculosis in the acquired immunodeficiency syndrome era. Am Rev Respir Dis 1991; 144: 1164-70.

37. Kim JH, Langston AA, Gallis HA. Miliary tuberculosis: epidemiology, clinical manifestations, diagnosis, and outcome. Rev Infect Dis 1990; 12: 583-90.

38. De Miguel, Encinar L, Villanueva R, García Rego J, Freire R, Diz Lois F. Tuberculosis miliar en adultos. Estudio en 67 pacientes. An Med Interna (Madrid) 1990; 7: 5-12.

39. Veiga González M, Riestra Martínez M, Fresno Forcelledo M, González González M, Ablanedo Ablanedo P, Herrero Zapatero A. Tuberculosis miliar. Estudio autópsico de 29 casos. An Med Interna (Madrid) 1995; $12: 17-20$ 